\title{
Classical and quantum spinor cosmology with signature change
}

\author{
B. Vakili, S. Jalalzadeh ${ }^{\dagger}$ and H. R. Sepangi ${ }^{\ddagger}$ \\ Department of Physics, Shahid Beheshti University, Evin, Tehran 19839, Iran
}

October 30, 2018

\begin{abstract}
We study the classical and quantum cosmology of a universe in which the matter source is a massive Dirac spinor field and consider cases where such fields are either free or self-interacting. We focus attention on the spatially flat Robertson-Walker cosmology and classify the solutions of the Einstein-Dirac system in the case of zero, negative and positive cosmological constant $\Lambda$. For $\Lambda<0$, these solutions exhibit signature transitions from a Euclidean to a Lorentzian domain. In the case of massless spinor fields it is found that signature changing solutions do not exist when the field is free while in the case of a self-interacting spinor field such solutions may exist. The resulting quantum cosmology and the corresponding Wheeler-DeWitt equation are also studied for both free and self interacting spinor fields and closed form expressions for the wavefunction of the universe are presented. These solutions suggest a quantization rule for the energy.
\end{abstract}

PACS numbers: 04.20.-q, 04.20.Gz

*email: b-vakili@cc.sbu.ac.ir

${ }^{\dagger}$ email: s-jalalzadeh@cc.sbu.ac.ir

‡email: hr-sepangi@cc.sbu.ac.ir 


\section{Introdution}

The study of cosmology has always been influenced by the choice of the matter field used to construct the energy-momentum tensor in Einstein field equations. The most widely used matter source has traditionally been the perfect fluid. However, the ubiquitous scalar field has also been playing an increasingly important role in more recent cosmological models as the matter source. This of course is not surprising since being a "scalar field" makes it somewhat easy to work with. One may also conceivably imagine a universe filled with massless or massive spinor fields as the matter source. Such cosmological models have seldom been studied in the literature, and more often than not, in the form of general formalisms. In general then, it would be fair to say that cosmologies with spinor fields as the matter source are the least studied scenarios.

A question of interest related to classical and quantum cosmological models is that of signature transition which has been attracting attention since the early 1980's. Traditionally, a feature in general relativity is that one usually fixes the signature of the space-time metric before trying to solve Einstein's field equations. However, there is no a priori reason for doing so and it is now well known that the field equations do not demand this property, that is, if one relaxes this condition one may find solutions to the field equations which when parameterized suitably, can either have Euclidean or Lorentzian signature.

The notion of signature transition mainly started to appear in the works of Hartle and Hawking [1] where they argued that in quantum cosmology amplitudes for gravity should be expressed as the sum of all compact Riemannian manifolds whose boundaries are located at the signature changing hypersurface. In more recent times, a number of authors have studied this problem when a scalar field is coupled to Einstein field equations and shown that the resulting solutions, when properly parameterized, exhibit signature transition, see for example [2]. In a similar vain, a classical model is studied in [3] in which a self-interacting scalar field is coupled to Einstein's equations with a Sinh-Gordon interaction potential. The field equations are then solved exactly for the scale factor and scalar field, giving rise to a spatially flat Robertson-Walker cosmology with signature changing properties. The case of the non-flat universe is addressed in [4] with a discussion about the conditions under which signature transition exists. Also, it is shown in [4] that in the case of a massless scalar field this phenomena does not exist. At the quantum cosmology level the same problem is investigated in [5] with an analysis pertaining to the exact solutions of the Wheeler-DeWitt equation. Signature transition has also been studied in multi-dimensional classical and quantum cosmology in [6] where a $4+d$-dimensional space-time is minimally coupled to a scalar field. Finally, it has been used as the compactification mechanism in Kaluza-Klein cosmology $[7,8]$ for a positive and negative cosmological constant respectively.

As a natural extension of the works done in [3] and [4] and as a specific example, we consider a spinor field as the matter source interacting with gravity and itself in a Robertson-Walker geometry. This is the subject of study in this paper. In general, studying spinor fields coupled to gravity results in Einstein-Dirac systems which are not easy to solve. The cosmological solutions of Einstein-Dirac systems have been studied in few cases by some authors, see [9] for example and the references therein. The fourth reference in [9] is notable in that the quantization of a spinor field coupled to gravity is studied in a Robetrson-Walker background. Here we analyze a classical model with spatially flat Robertson-Walker cosmology in which a massive spinor field is coupled to gravity. We show that in this model signature transition occurs only if the cosmological constant is negative. We also show that a massless free spinor field does not lead to signature transition while a massless self-interacting spinor field does.

The quantum cosmology of this model, embodied in the solutions of the corresponding WheelerDeWitt (WD) equation is studied for both free and self interacting spinor fields. It turns out that the WD equation posses exact solutions in terms of Hypergeometric functions. Also, the "zero energy condition" imposes a quantization condition on the solutions for the spinor fields. These wavefunctions correspond to the classical solutions undergoing signature transition from a Euclidean to a Lorentzian 
domain and, as such, could be useful in understanding the initial condition of the universe.

The paper is organized as follows: in section 2 we write the Dirac equation in spatially flat Robertson-Walker background. Section 3 deals with the energy-momentum tensor of the spinor field and the Einstein-Dirac field equations. In section 4 we classify the exact solutions in the cases of a free and self-interacting spinor field when the cosmological constant is zero, negative or positive respectively and investigate signature transition in each case. The effect of a massless spinor field on signature transition is studied in section 5. Section 6 is devoted to the study of the quantum cosmology of our model and finally, conclusions are drawn in section 7 .

\section{Dirac equation}

Since our aim is to solve the coupled Einstein-Dirac equations in a cosmological background, let us concentrate on writing the Dirac equation in a spatially flat Robertson-Walker space-time given by

$$
d s^{2}=-d t^{2}+R^{2}(t)\left(d x^{2}+d y^{2}+d z^{2}\right) .
$$

As is well known the Dirac equation in curved space-time can be obtained from the Lagrangian $(c=\hbar=1)$

$$
L=\frac{1}{2}\left[\bar{\psi} \gamma^{\mu}\left(\partial_{\mu}+\Gamma_{\mu}\right) \psi-\bar{\psi}\left(\overleftarrow{\partial_{\mu}}-\Gamma_{\mu}\right) \gamma^{\mu} \psi\right]-V(\bar{\psi}, \psi)
$$

where $V(\bar{\psi}, \psi)$ is a potential describing the interaction of the spinor field $\psi$ with itself, $\gamma^{\mu}$ are the Dirac matrices associated with the spacetime metric satisfying $\left\{\gamma^{\mu}, \gamma^{\nu}\right\}=2 g^{\mu \nu}$ and $\Gamma_{\mu}$ are the spin connections. The Euler-Lagrange equations for $\psi$ and $\bar{\psi}$ then yield

$$
\begin{aligned}
& \gamma^{\mu}\left(\partial_{\mu}+\Gamma_{\mu}\right) \psi-\frac{\partial V}{\partial \bar{\psi}}=0 \\
& \bar{\psi}\left(\overleftarrow{\partial_{\mu}}-\Gamma_{\mu}\right) \gamma^{\mu}+\frac{\partial V}{\partial \psi}=0
\end{aligned}
$$

The $\gamma^{\mu}$ matrices are related to the flat Dirac matrices, $\gamma^{a}$, through the tetrads $e_{\mu}^{a}$ as follows

$$
\gamma^{\mu}=e_{a}^{\mu} \gamma^{a}, \quad \gamma_{\mu}=e_{\mu}^{a} \gamma_{a}
$$

For metric (1) the tetrads can be easily obtained from their definitions, that is $g_{\mu \nu}=e_{\mu}^{a} e_{\nu}^{b} \eta_{a b}$, leading to

$$
e_{\mu}^{a}=\operatorname{diag}(1, R, R, R), \quad e_{a}^{\mu}=\operatorname{diag}(1,1 / R, 1 / R, 1 / R) .
$$

Also, the spin connections satisfy the relation

$$
\Gamma_{\mu}=\frac{1}{4} g_{\nu \lambda}\left(\partial_{\mu} e_{a}^{\lambda}+\Gamma_{\sigma \mu}^{\lambda} e_{a}^{\sigma}\right) \gamma^{\nu} \gamma^{a} .
$$

Thus, for the line element (1), use of (5) and (6) yeilds

$$
\Gamma_{0}=0, \quad \Gamma_{i}=-\frac{\dot{R}}{2} \gamma^{0} \gamma^{i},
$$

where $\gamma^{0}$ and $\gamma^{i}$ are the Dirac matrices in the Minkowski space and we have adapted the following representation

$$
\gamma^{0}=\left(\begin{array}{cc}
-i & 0 \\
0 & i
\end{array}\right), \quad \gamma^{i}=\left(\begin{array}{cc}
0 & \sigma^{i} \\
\sigma^{i} & 0
\end{array}\right)
$$


The preliminary setup is now complete for writing the Dirac equation. From equations (3) and (4) we have

$$
\begin{aligned}
& {\left[\frac{\partial}{\partial t}-\frac{1}{R} \gamma^{0}\left(\gamma^{1} \frac{\partial}{\partial x}+\gamma^{2} \frac{\partial}{\partial y}+\gamma^{3} \frac{\partial}{\partial z}\right)+\frac{3}{2} \frac{\dot{R}}{R}\right] \psi+\gamma^{0} \frac{\partial V}{\partial \bar{\psi}}=0} \\
& \bar{\psi}\left[\frac{\overleftarrow{\partial}}{\partial t}-\frac{1}{R}\left(\frac{\overleftarrow{\partial}}{\partial x} \gamma^{1}+\frac{\overleftarrow{\partial}}{\partial y} \gamma^{2}+\frac{\overleftarrow{\partial}}{\partial z} \gamma^{3}\right) \gamma^{0}+\frac{3}{2} \frac{\dot{R}}{R}\right]-\frac{\partial V}{\partial \psi} \gamma^{0}=0
\end{aligned}
$$

Since the metric functions in (1) are functions of $t$ only, we may write $\psi(\mathbf{r}, t)=e^{i \mathbf{p} \cdot \mathbf{r}} \psi(t)$ in (10) where $\mathbf{p}=\left(p_{1}, p_{2}, p_{3}\right)$ are the separation constants.

The energy-momentum tensor for the Dirac spinor field as a matter source for gravity can be obtained from the definition

$$
T_{\mu \nu}=2 \frac{\partial L}{\partial g^{\mu \nu}}-g_{\mu \nu} L
$$

which results in

$$
T_{\mu \nu}=\frac{1}{2}\left[\bar{\psi} \gamma_{(\mu} \nabla_{\nu)} \psi-\left(\nabla_{(\mu} \bar{\psi}\right) \gamma_{\nu)} \psi\right]-g_{\mu \nu} L
$$

where

$$
\nabla_{\mu} \psi=\left(\partial_{\mu}+\Gamma_{\mu}\right) \psi \quad \text { and } \quad \nabla_{\mu} \bar{\psi}=\bar{\psi}\left(\overleftarrow{\partial_{\mu}}-\Gamma_{\mu}\right)
$$

Since the Einstein tensor associated with metric (1) is diagonal, the energy-momentum tensor of its matter source should also be diagonal. With the anzats $\psi(\mathbf{r}, t)=e^{i \mathbf{p} \cdot \mathbf{r}} \psi(t)$ the off-diagonal components of $T_{\mu \nu}$ become

$$
T_{i j}=\frac{1}{2}\left(p_{i} \bar{\psi} \gamma_{j} \psi+p_{j} \bar{\psi} \gamma_{i} \psi\right) \quad \text { and } \quad T_{0 j}=-p_{j} \bar{\psi} \gamma_{0} \psi
$$

and hence for them to vanish we must have $p_{i}=0$, i.e. the spinor field $\psi$ depends on $t$ only. Therefore equations (10) and (11) simplify to

$$
\begin{aligned}
& \frac{d \psi}{d t}+\frac{3}{2} \frac{\dot{R}}{R} \psi+\gamma^{0} \frac{\partial V}{\partial \bar{\psi}}=0, \\
& \frac{d \bar{\psi}}{d t}+\frac{3}{2} \frac{\dot{R}}{R} \bar{\psi}-\frac{\partial V}{\partial \psi} \gamma^{0}=0 .
\end{aligned}
$$

The components of the energy-momentum tensor can then be evaluated from equations (13) and (14) with the result

$$
T_{0}^{0}=V(\bar{\psi}, \psi), \quad T_{1}^{1}=T_{2}^{2}=T_{3}^{3}=-\frac{1}{2}\left(\bar{\psi} \frac{\partial V}{\partial \bar{\psi}}+\frac{\partial V}{\partial \psi} \psi\right)+V(\bar{\psi}, \psi)
$$

\section{$3 \quad$ Field equations}

In this section we consider the Einstein-Dirac equation written as

$$
G_{\mu \nu}=R_{\mu \nu}-\frac{1}{2} \mathcal{R} g_{\mu \nu}+\Lambda g_{\mu \nu}=\kappa T_{\mu \nu}(\bar{\psi}, \psi)
$$

where $R_{\mu \nu}, \mathcal{R}$ and $\Lambda$ are Ricci tensor, scalar curvature and cosmological constant respectively, constructed from torsion-free connections compatible with the metric. The spinor field $\psi$ which interacts with itself through the potential $V(\bar{\psi}, \psi)$ is a solution of the Dirac equation (14) and is coupled to gravity with the energy-momentum tensor given by equation (13). These coupled Einstein-Dirac equations must now be solved in a domain that would lead to a spatially flat Robertson-Walker cosmology given by equation (1) with Lorentzian signature $(-,+,+,+)$. However we may parameterize the metric in such a way as to allow solutions with continuous transition to a Euclidean domain. To 
this end, we parameterize the metric, as in [3] by adapting the chart $\{\beta, x, y, z\}$ where the hypersurface of signature change would be characterized by $\beta=0$. The metric can then be parameterized in terms of the scale function $R(\beta)$ and the lapse function $\beta$ to take the form

$$
d s^{2}=-\beta d \beta^{2}+R^{2}(\beta)\left(d x^{2}+d y^{2}+d z^{2}\right) .
$$

It is now clear that the sign of $\beta$ determines the geometry, being Lorentzian if $\beta>0$ and Euclidean if $\beta<0$. For $\beta>0$, the traditional cosmic time can be recovered by the substitution $t=\frac{2}{3} \beta^{3 / 2}$. Adapting the chart $\{t, x, y, z\}$, we shall write $R(t)=R(\beta(t))$ and also $\psi(t)=\psi(\beta(t))$. Using equations (14) through (18) with units in which $\kappa=\hbar=c=1$ one finds the following differential equations

$$
\begin{gathered}
3\left(\frac{\dot{R}}{R}\right)^{2}-\Lambda=-V(\bar{\psi}, \psi), \\
2\left(\frac{\dot{R}}{R}\right)+3\left(\frac{\dot{R}}{R}\right)^{2}-\Lambda=\frac{1}{2}\left(\bar{\psi} \frac{\partial V}{\partial \bar{\psi}}+\frac{\partial V}{\partial \psi} \psi\right)-V(\bar{\psi}, \psi), \\
\dot{\psi}+\frac{3}{2} \frac{\dot{R}}{R} \psi+\gamma^{0} \frac{\partial V}{\partial \bar{\psi}}=0, \\
\dot{\bar{\psi}}+\frac{3}{2} \frac{\dot{R}}{R} \bar{\psi}-\frac{\partial V}{\partial \psi} \gamma^{0}=0,
\end{gathered}
$$

where a dot represents differentiation with respect to $t$. We formulate our differential equations in a region that does not include $\beta=0$ and seek solutions for $R$ and $\psi$ that smoothly pass through the $\beta=0$ hypersurface. The scalar curvature corresponding to our metric is

$$
\mathcal{R}=6\left[\frac{\ddot{R}}{R}+\left(\frac{\dot{R}}{R}\right)^{2}\right],
$$

while the energy density of the spinor field is given by

$$
\rho=T_{00}=-V(\bar{\psi}, \psi)
$$

It should be noted that equations (19)-(22) are not all independent. We shall see that with a suitable choice for the potential $V(\bar{\psi}, \psi)$, equation (20) can be obtained by combining equations (19), (21) and (22). Also equation (22) is the Dirac equation for the adjoint spinor $\bar{\psi}$ and should not be considered as an independent equation. Thus, it is sufficient to solve either equations $(19,21)$ or equations $(20$, 21) which we shall endeavor to do in the next section after choosing a suitable form for the potential $V(\bar{\psi}, \psi)$.

\section{Solutions}

As mentioned in the last section integrability of the Einstein-Dirac field equations (19)-(22) depends on the choice of a suitable form for $V(\bar{\psi}, \psi)$. However, this potential should also describe a physical self-interacting spinor field. The potential is usually an invariant function constructed from the spinor $\psi$ and its adjoint $\bar{\psi}$. In general, difficulties in treating Einstein-Dirac systems depend directly on the form of the spinor $\psi$ and the potential $V(\bar{\psi}, \psi)$. For simplifying and addressing these difficulties we first demand that $\psi$ should be a function of $t$ only. This simplifies the Dirac equation (10) into the more manageable form (14). We should now choose a suitable self-interacting form for the potential. Some of the common forms for $V$ are: $V(\bar{\psi}, \psi)=m \bar{\psi} \psi$ representing a free spinor field of mass $m$, $V(\bar{\psi}, \psi)=m \bar{\psi} \psi+J_{\mu} J^{\mu}$ where $J^{\mu}=\bar{\psi} \gamma^{\mu} \psi$ and known as the Thirring model, $V(\bar{\psi}, \psi)=m \bar{\psi} \psi+(\bar{\psi} \psi)^{2}$ called Gross-Neveu model and $V(\bar{\psi}, \psi)=m \bar{\psi} \psi+(\bar{\psi} \psi)^{2}-\left(\bar{\psi} \gamma^{5} \psi\right)^{2}$ also known as the chiral GrossNeveu model [10]. Since in this work we are going to study signature transition for the solutions 
of the problem at hand, we concentrate on the two of the simplest forms for $V(\bar{\psi}, \psi)$ which either give the exact solutions of the field equations or show signature transition effects. We therefore focus attention on the free spinor field and self-interacting spinor field potentials with the latter having a term of the form $(\bar{\psi} \psi)^{2}$.

\subsection{Free spinor field}

This is the case in which the potential has the form $V(\bar{\psi}, \psi)=m \bar{\psi} \psi$. From equation (16) the components of the energy-momentum tensor are $T_{0}^{0}=m \bar{\psi} \psi$ and $T_{i}^{i}=0$, and our field equations become

$$
\begin{gathered}
3\left(\frac{\dot{R}}{R}\right)^{2}-\Lambda=-m \bar{\psi} \psi \\
2\left(\frac{\dot{R}}{R}\right)^{\cdot}+3\left(\frac{\dot{R}}{R}\right)^{2}-\Lambda=0 \\
\dot{\psi}+\frac{3}{2} \frac{\dot{R}}{R} \psi+m \gamma^{0} \psi=0 \\
\dot{\bar{\psi}}+\frac{3}{2} \frac{\dot{R}}{R} \bar{\psi}-m \bar{\psi} \gamma^{0}=0
\end{gathered}
$$

The solutions of this system can easily be obtained. The results in terms of $\beta$ are listed as follows:

Case (a) $\Lambda=0$ : in this case integrating equations (25)-(28) results in the solutions

$$
\begin{gathered}
R(\beta)=\left(M \beta^{3 / 2}+A\right)^{2 / 3}, \\
\psi=\left(\begin{array}{c}
\psi_{+} \\
\psi_{-}
\end{array}\right), \quad \psi_{ \pm} \sim \frac{e^{ \pm 2 / 3 i m \beta^{3 / 2}}}{M \beta^{3 / 2}+A} .
\end{gathered}
$$

Also the scalar curvature $\mathcal{R}$ and energy density $\rho$ of the spinor field are found to be

$$
\mathcal{R}=\frac{3 M^{2}}{\left(M \beta^{3 / 2}+A\right)^{2}}, \quad \rho=\frac{3 M^{2}}{\left(M \beta^{3 / 2}+A\right)^{2}},
$$

where $M$ (being proportional to $m$ ) and $A$ are integrating constants. We see that if $A \neq 0$, the scale factor $R(\beta)$ is not a real function in the Euclidean region $\beta<0$. If $A=0$ then $R(\beta) \sim \beta$ is an

unbounded function in both Euclidean and Lorentzian regions, passing continuously through $\beta=0$. However, in this case the functions $\psi_{ \pm}, \mathcal{R}$ and $\rho$ all have a singular behavior in $\beta=0$ but are well defined in the domains $\beta>0$ and $\beta<0$. The solutions in the case of zero cosmological constant are therefore not suitable candidates for exhibiting signature transition behavior.

Case (b) $\Lambda<0$ : in this case the solutions can be written as

$$
\begin{gathered}
R(\beta)=\left(\frac{M}{-\Lambda}\right)^{1 / 3} \cos ^{2 / 3}\left(\frac{\sqrt{-3 \Lambda}}{3} \beta^{3 / 2}\right) \\
\psi_{ \pm} \sim \frac{e^{ \pm 2 / 3 i m \beta^{3 / 2}}}{\sqrt{\frac{M}{-\Lambda}} \cos \left(\frac{\sqrt{-3 \Lambda}}{3} \beta^{3 / 2}\right)} \\
\mathcal{R}=-\Lambda\left[\tan ^{2}\left(\frac{\sqrt{-3 \Lambda}}{3} \beta^{3 / 2}\right)-3\right], \quad \rho=\frac{-\Lambda}{\cos ^{2}\left(\frac{\sqrt{-3 \Lambda}}{3} \beta^{3 / 2}\right)},
\end{gathered}
$$



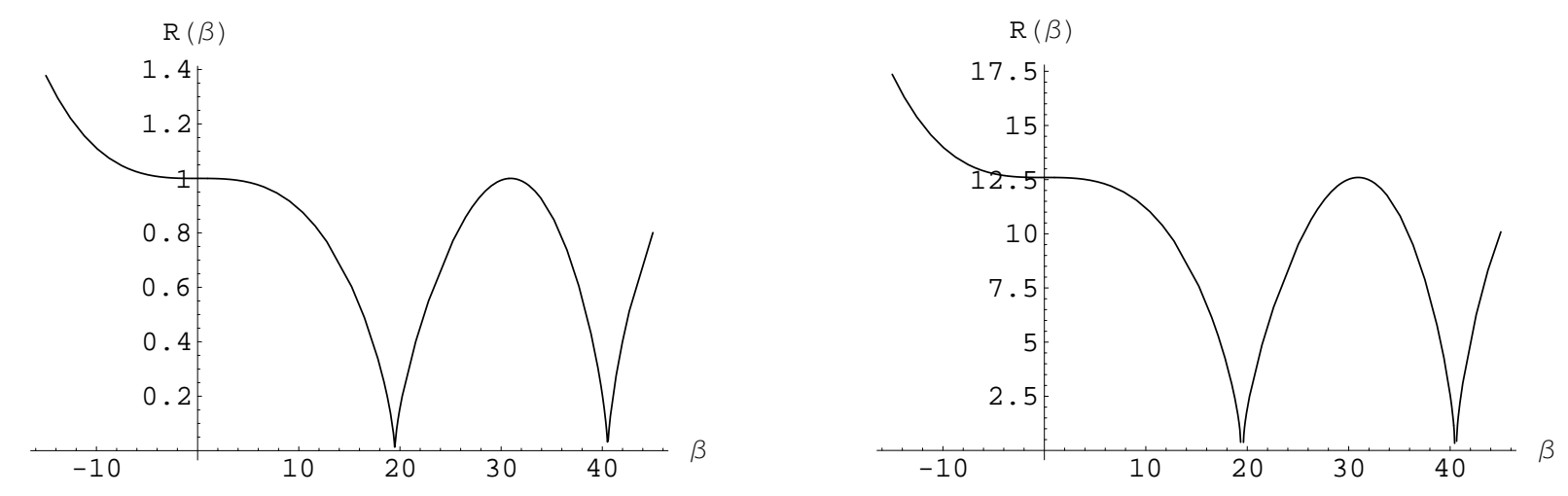

Figure 1: Left, the scale factor for a free spinor field and right, the same figure for a self interacting spinor field, both for a typical negative value of $\Lambda=-10^{-3}$ with $M=g=1$.
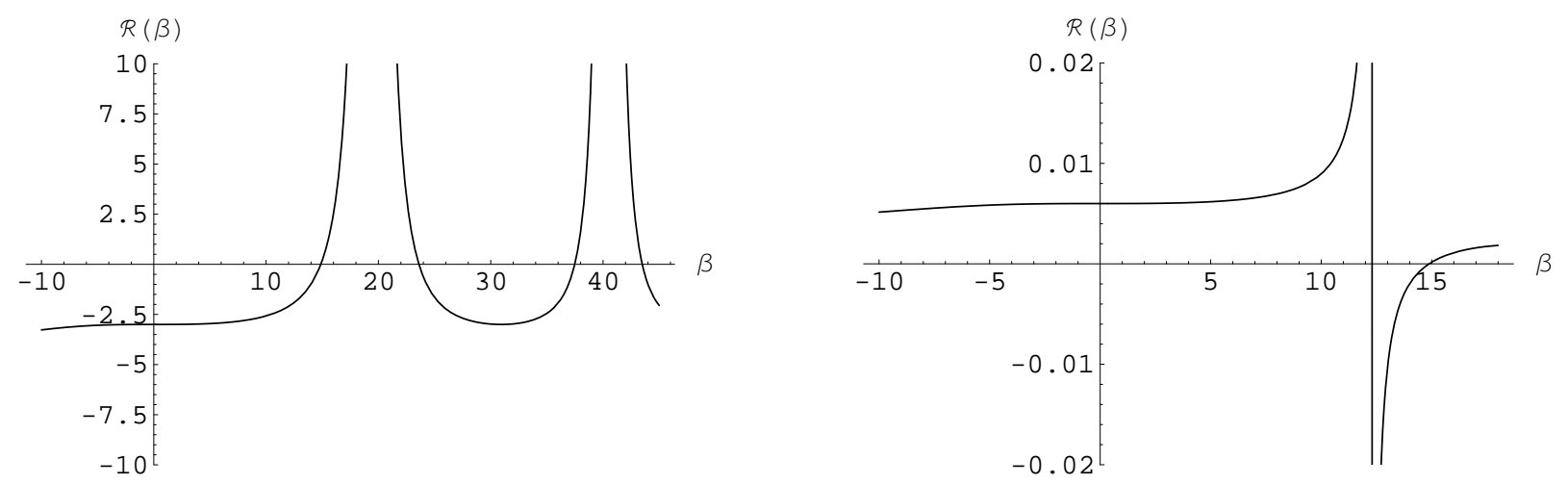

Figure 2: Left, the curvature scalar for a free spinor field and right, the same figure for a self interacting spinor field, both for a typical negative value of $\Lambda=-10^{-3}$ with $M=g=1$.

where $M$ is an integrating constant proportional to $m$, the mass of the spinor field. The other integrating constant is chosen so that $\dot{R}(\beta=0)=0$. The scale factor $R(\beta)$ in this case is a real function and behaves exponentially for $\beta<0$, passing smoothly through $\beta=0$ and becomes a bounded oscillatory function for $\beta>0$. Also, the physical quantities $\mathcal{R}, \psi_{ \pm}$and $\rho$ are regular functions both in the Euclidean and Lorentzian domains and pass continuously through the $\beta=0$ hypersurface. Thus all of the above results show that the solutions in the case of a negative cosmological constant exhibit signature transition from a Euclidean to a Lorentzian domain. A plot of the scale factor, scalar curvature and energy density in terms of $\beta$ are shown in figure 1 through 3 , using equations (32) and (34).

Case (c) $\Lambda>0$ : in this case for integrating the system of equations (25)-(28) we must compare the Hubble parameter $H=\frac{\dot{R}}{R}$ with the value of $\sqrt{\frac{\Lambda}{3}}$. If $H^{2}>\frac{\Lambda}{3}$, the solutions of the field equations in terms of the parameter $\beta$ read

$$
R(\beta)=\left(\frac{M}{\Lambda}\right)^{1 / 3} \sinh ^{2 / 3}\left(\frac{\sqrt{3 \Lambda}}{3} \beta^{3 / 2}+A\right),
$$

where $M$, again proportional to the mass of spinor field $m$, and $A$ are integrating constants. If we take $A=0$, the scale factor $R(\beta)$ shows regular behavior in $\beta<0$ and $\beta>0$ regions and also pass smoothly through $\beta=0$. However, the scalar curvature, energy density and spinor field

$$
\mathcal{R}=\Lambda\left[\operatorname{coth}^{2}\left(\frac{\sqrt{3 \Lambda}}{3} \beta^{3 / 2}\right)-3\right],
$$



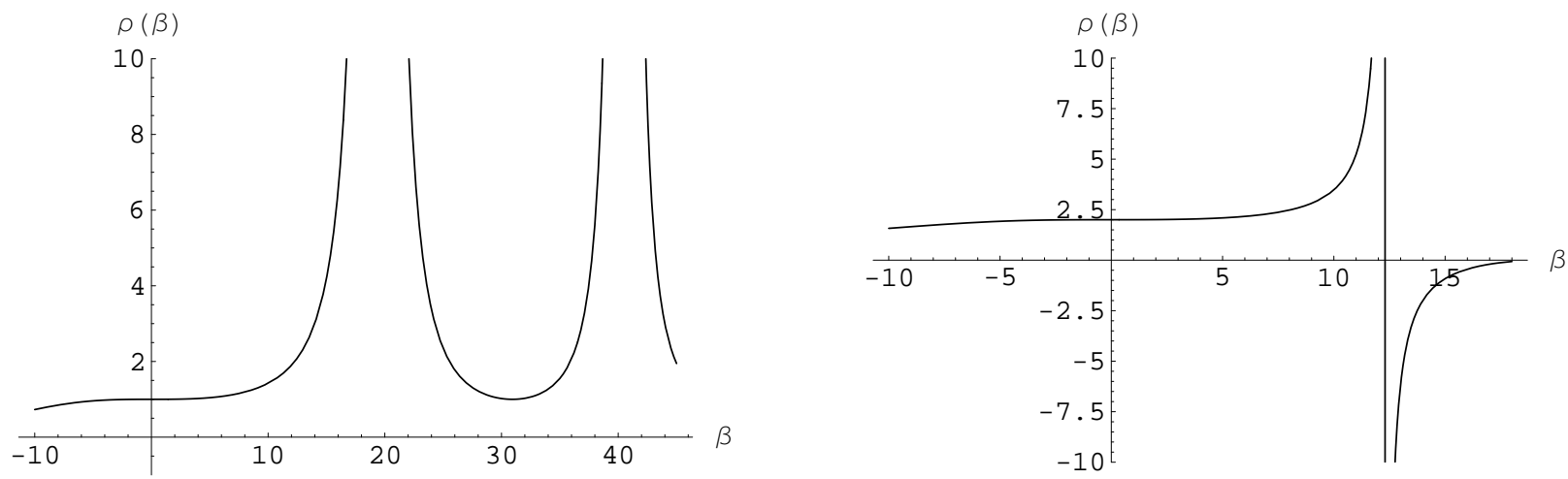

Figure 3: Left, the energy density for a free spinor field and right, the same figure for a self interacting spinor field, both for a typical negative value of $\Lambda=-10^{-3}$ with $M=g=1$.

$$
\begin{gathered}
\rho=\frac{\Lambda}{\sinh ^{2}\left(\frac{\sqrt{3 \Lambda}}{3} \beta^{3 / 2}\right)}, \\
\psi_{ \pm} \sim \frac{e^{ \pm 2 / 3 i m} \beta^{3 / 2}}{\sinh \left(\frac{\sqrt{3 \Lambda}}{3} \beta^{3 / 2}\right)},
\end{gathered}
$$

all have singularity a at $\beta=0$. In the case $A \neq 0$ the scale factor (35) is not a well defined real function when passing through $\beta=0$. If $H^{2}<\frac{\Lambda}{3}$, the above solutions take the form

$$
\begin{gathered}
R(\beta)=\left(\frac{M}{\Lambda}\right)^{1 / 3} \cosh ^{2 / 3}\left(\frac{\sqrt{3 \Lambda}}{3} \beta^{3 / 2}\right), \\
\mathcal{R}=\Lambda\left[\tanh ^{2}\left(\frac{\sqrt{3 \Lambda}}{3} \beta^{3 / 2}\right)+3\right] \\
\rho=\frac{-\Lambda}{\cosh ^{2}\left(\frac{\sqrt{3 \Lambda}}{3} \beta^{3 / 2}\right)} \\
\psi_{ \pm} \sim \frac{e^{ \pm 2 / 3 i m} \beta^{3 / 2}}{\cosh \left(\frac{\sqrt{3 \Lambda}}{3} \beta^{3 / 2}\right)}
\end{gathered}
$$

where we have taken the integrating constant $A=0$. These solutions resemble the ones for the case $\Lambda<0$ and exhibit signature transition. However, we should note that the energy density of the spinor field is negative and thus unphysical. In summery, the above discussion shows that within the context of this model, a universe with positive cosmological constant cannot undergo signature transition from a Euclidean to a Lorentzian domain through the $\beta=0$ hypersurface where its matter source is a massive free spinor field.

\subsection{Self-interacting spinor field}

In this section we return to the field equations (19)-(22) with a potential describing a spinor field interacting with itself. The form which we have chosen for this potential is that of the Gross-Neveu model

$$
V(\bar{\psi}, \psi)=m \bar{\psi} \psi+\lambda(\bar{\psi} \psi)^{2},
$$

where $\lambda$ is a coupling constant. From (16) the components of the energy-momentum tensor read

$$
T_{0}^{0}=m \bar{\psi} \psi+\lambda(\bar{\psi} \psi)^{2}, \quad T_{1}^{1}=T_{2}^{2}=T_{3}^{3}=-\lambda(\bar{\psi} \psi)^{2} .
$$


The field equations (19)-(22) can now be written as

$$
\begin{aligned}
& 3\left(\frac{\dot{R}}{R}\right)^{2}-\Lambda=-m \bar{\psi} \psi-\lambda(\bar{\psi} \psi)^{2}, \\
& 2\left(\frac{\dot{R}}{R}\right)^{\cdot}+3\left(\frac{\dot{R}}{R}\right)^{2}-\Lambda=\lambda(\bar{\psi} \psi)^{2}, \\
& \dot{\psi}+\frac{3}{2} \frac{\dot{R}}{R} \psi+(m+2 \lambda \bar{\psi} \psi) \gamma^{0} \psi=0, \\
& \dot{\bar{\psi}}+\frac{3}{2} \frac{\dot{R}}{R} \bar{\psi}-(m+2 \lambda \bar{\psi} \psi) \bar{\psi} \gamma^{0}=0 .
\end{aligned}
$$

If $\Lambda=0$, the scale factor as a function of $\beta$ obtained from the above equations reads

$$
R(\beta)=\left(M \beta^{3}-g\right)^{1 / 3},
$$

where $M$ and $g$ are two constants proportional to the mass of the spinor field and the coupling constant $\lambda$ respectively. The other integrating constant is taken to be zero. This function shows an unbounded scale factor both in the Euclidean and Lorentzian domains which passes continuously through $\beta=0$. The scalar curvature and the energy density of the spinor field are found to be

$$
\mathcal{R}=-\frac{1}{3} M \frac{M \beta^{3}-3 g}{\left(M \beta^{3}-g\right)^{2}}, \quad \rho=\frac{3 M^{2} \beta^{3}}{\left(M \beta^{3}-g\right)^{2}} .
$$

Functionally speaking, these are well defined in both $\beta<0$ and $\beta>0$ regions, passing smoothly through the signature changing hypersurface. However, the question arises as to the validity of these solutions since the energy density is again negative in the Euclidean region $(\beta<0)$, pointing to an unphysical matter source. Like the free spinor field case, when $\Lambda>0$, we are not led to physically well defined solutions. In the case of $\Lambda<0$ the scale factor, being obtained from the system of equations (45)-(48) can be written as

$$
R(\beta)=\left[\frac{M}{-\Lambda}+\sqrt{\frac{M^{2}}{\Lambda^{2}}+\frac{g}{-\Lambda}} \cos \left(\frac{2}{3} \sqrt{-3 \Lambda} \beta^{3 / 2}\right)\right]^{1 / 3},
$$

where as before $M$ and $g$ are equal to $m$ and $\lambda$ up to an integrating constant, and the other integrating constant is choose so that $\dot{R}(\beta=0)=0$. Also the Ricci scalar and the spinor field energy density are obtained as follows

$$
\begin{aligned}
& \mathcal{R}=\frac{6}{\frac{M}{-\Lambda}+\sqrt{\frac{M^{2}}{\Lambda^{2}}+\frac{g}{-\Lambda}} \cos \left(\frac{2}{3} \sqrt{-3 \Lambda} \beta^{3 / 2}\right)} \\
& \times {\left[\sqrt{M^{2}-g \Lambda} \cos \left(\frac{2}{3} \sqrt{-3 \Lambda} \beta^{3 / 2}\right)+\frac{\left(\frac{M^{2}}{-3 \Lambda}+\frac{g}{3}\right) \sin ^{2}\left(\frac{2}{3} \sqrt{-3 \Lambda} \beta^{3 / 2}\right)}{\frac{M}{-\Lambda}+\sqrt{\frac{M^{2}}{\Lambda^{2}}+\frac{g}{-\Lambda}} \cos \left(\frac{2}{3} \sqrt{-3 \Lambda} \beta^{3 / 2}\right)}\right] } \\
& \rho=\frac{1}{\frac{M}{-\Lambda}+\sqrt{\frac{M^{2}}{\Lambda^{2}}+\frac{g}{-\Lambda}} \cos \left(\frac{2}{3} \sqrt{-3 \Lambda} \beta^{3 / 2}\right)}\left[2 M+\frac{g}{\frac{M}{-\Lambda}+\sqrt{\frac{M^{2}}{\Lambda^{2}}+\frac{g}{-\Lambda}} \cos \left(\frac{2}{3} \sqrt{-3 \Lambda} \beta^{3 / 2}\right)}\right] .
\end{aligned}
$$

A quick look at the functions (51)-(53) shows their regular behavior both in the Euclidean and Lorentzian regions without any singularity at $\beta=0$. From (51) it is clear that the corresponding cosmology is an unbounded universe in the Euclidean domain having a bounded oscillatory behavior in the Lorentzian region and passes smoothly through the hypersurface of signature transition at $\beta=0$. Figure 1 through 3 show plots of the scale factor, scalar curvature and energy density using equations (51), (52) and (53). 


\section{$5 \quad$ Massless spinor field}

At this point it would be interesting to take the massless limit of the pervious results. If $m=0$ and the spinor field is free, i.e. $V(\bar{\psi}, \psi)=m \bar{\psi} \psi$, then from equations (25)-(28) we obtain $R(\beta)=$ $e^{\frac{2}{3} c \beta^{3 / 2}}$, where $c$ is a constant. This is not a real function for $\beta<0$ and thus does not exhibit signature transition. In the case of a self-interacting spinor field however, the massless limit can be obtained easily by substituting $M=0$ in equations (51)-(53), which again results in signature changing solutions. To summarize, solutions exhibiting signature transition do not exist when the spinor field is free and massless.

\section{Quantum cosmology}

The study of quantum cosmology of the model presented above is the goal we shall pursue in this section. For this purpose we construct the Hamiltonian of our model. Let us start with the actin

$$
\mathcal{S}=\int \sqrt{-g}\left(L_{\text {grav }}+L_{\text {matt }}\right) d^{4} x
$$

where

$$
L_{\text {grav }}=\mathcal{R}-\Lambda,
$$

is the Einstein-Hilbert Lagrangian for the gravitational field and $L_{\text {matt }}$ represents the Lagrangian of the matter source which is given by (2) with $g$ being the determinant of the metric here. Now, by substituting (2) and (55) in (54) and integrating over spatial dimensions, we are led to an effective Lagrangian in the mini-superspace $\{R, \psi, \bar{\psi}\}$ as follows

$$
\mathcal{L}=R \dot{R}^{2}+\frac{1}{3} \Lambda R^{3}+\frac{1}{6} R^{3}\left[\bar{\psi} \gamma^{0} \dot{\psi}-\dot{\bar{\psi}} \gamma^{0} \psi-2 V(\bar{\psi}, \psi)\right]
$$

Variation of the above Lagrangian with respect to $R, \bar{\psi}$ and $\psi$ yields equations (20), (21) and (22) respectively. Also, we have the "zero energy condition" given by

$$
\mathcal{H}=\frac{\partial \mathcal{L}}{\partial \dot{R}} \dot{R}+\frac{\partial \mathcal{L}}{\partial \dot{\psi}} \dot{\psi}+\dot{\bar{\psi}} \frac{\partial \mathcal{L}}{\partial \dot{\bar{\psi}}}-\mathcal{L}=0
$$

which yields the constraint equation (19). To simplify the Lagrangian (56), consider the change of variable $u=R^{3 / 2}$. In terms of this new variable Lagrangian (56) takes the form

$$
\mathcal{L}=\frac{4}{9} \dot{u}^{2}+\frac{1}{3} \Lambda u^{2}+\frac{1}{6} u^{2}\left[\bar{\psi} \gamma^{0} \dot{\psi}-\dot{\bar{\psi}} \gamma^{0} \psi-2 V(\bar{\psi}, \psi)\right]
$$

with the corresponding Hamiltonian given by

$$
\mathcal{H}=\frac{4}{9} \dot{u}^{2}-\frac{1}{3} \Lambda u^{2}+\frac{1}{3} u^{2} V(\bar{\psi}, \psi)=0 .
$$

Choosing the potential $V(\bar{\psi}, \psi)=m \bar{\psi} \psi+\lambda(\bar{\psi} \psi)^{2}$ and noting that equations (47) and (48) allow us to

write $\bar{\psi} \psi=\frac{\mathcal{C}}{R^{3}}=\frac{\mathcal{C}}{u^{2}}$ and substitute for the terms involving $\dot{\psi}$ and $\dot{\bar{\psi}}$, we construct the Wheeler-DeWitt equation from Hamiltonian constraint (59) with the result

$$
\mathcal{H} \Psi(u)=\left[\frac{d^{2}}{d u^{2}}-\omega^{2} u^{2}+M-\frac{g}{u^{2}}\right] \Psi(u)=0,
$$

where $\omega^{2}=-\frac{16}{27} \Lambda, M=\frac{16}{27} m, g=\frac{16}{27} \lambda$ and $\Psi(u)$ is the wave function of the universe. Note that we have taken $\mathcal{C}=-1$ to obtain a positive energy density for the spinor field, since in the case of a 
positive cosmological constant, our classical solutions (35-42) do not exhibit a physical situation. In what follows, we only consider the case where $\Lambda<0$.

In order to solve equation (60) we first consider the case $g=0$, which is relevant to the free spinor field. In this case equation (60) becomes to a Hermite equation and its eigenfunctions can be written in terms of Hermite polynomials $H_{n}(x)$ as

$$
\Phi_{n}(u)=\left(\frac{\omega}{\pi}\right)^{1 / 4} \frac{1}{\sqrt{2^{n} n !}} e^{-\omega u^{2} / 2} H_{n}(\sqrt{\omega} u), \quad n=0,1,2, \cdots
$$

The "zero energy condition" $\mathcal{H}=0$ then yields

$$
m=\frac{27}{16}(2 n+1) \omega .
$$

We note that the energy density of the spinor field in this case is $\rho=-m \bar{\psi} \psi=\frac{m}{R^{3}}$. Thus, in our units, $m$ is the total energy of the spinor field. Therefore, equation (62) is the quantization condition of the total energy of the spinor field. The general solution of the WD equation in this case can be written as

$$
\Psi(u)=\sum_{n}^{\prime} c_{n} \Phi_{n}(u)
$$

where the prime on the sum indicates summing over all values of $n$ satisfying condition (62).

For a self-interacting spinor field, that is for $g \neq 0$, equation (60) after a change of variable $v=\omega u^{2}$ and transformation $\psi=v^{-1 / 4} \phi$ becomes

$$
\frac{d^{2} \phi}{d v^{2}}+\left(-\frac{1}{4}+\frac{\kappa}{v}+\frac{1 / 4-\mu^{2}}{v^{2}}\right) \phi=0,
$$

where $\kappa=\frac{M}{4 \omega}$ and $\mu^{2}=\frac{1}{16}+\frac{g}{4}$. The above equation is the Whittaker differential equation and its solution can be written in terms of confluent Hypergeometric functions $M(a, b, x)$ and $U(a, b, x)$ as

$$
\phi(v)=e^{-v / 2} v^{\mu+1 / 2}\left[c_{1} M\left(\mu-\kappa+\frac{1}{2}, 2 \mu+1 ; v\right)+c_{2} U\left(\mu-\kappa+\frac{1}{2}, 2 \mu+1 ; v\right)\right] .
$$

Bearing in the mind that if $g=0$ the self-interacting solution should be reduced to solution (61), we take $c_{1}=0$ and retain only the functions $U(a, b, x)[11]$. Therefore, the wave function $\Psi(u)$ is equal to

$$
\Psi(u)=\left(\omega u^{2}\right)^{\frac{1+\sqrt{1+4 g}}{4}} e^{-\omega u^{2} / 2} U\left(\frac{1}{2}-\frac{M}{4 \omega}+\frac{\sqrt{1+4 g}}{4}, 1+\frac{\sqrt{1+4 g}}{2} ; \omega u^{2}\right) .
$$

A glance at equation (60) shows that the solutions have a behavior of the form $\Psi \sim e^{-\omega u^{2} / 2}$ when $u \rightarrow \infty$ and $\Psi \sim\left(u^{2}\right)^{(1+\sqrt{1+4 g}) / 4}$ in the limit where $u \rightarrow 0$. Demanding the same limiting behavior for solutions (66), one can easily see that the function $U(a, b, x)$ in (66) should reduce to a polynomial and this happens when $a=-n$ [11]. This yields a quantization condition for the total energy of the self interacting spinor field as follows

$$
m=\frac{27}{8}\left(2 n+1+\frac{\sqrt{1+4 g}}{2}\right) .
$$

Figure 4 shows $|\Psi(u)|^{2}$ for typical values of the parameters. As it is clear from this figure, the wavefunction has a well defined behavior near $u=0$ and describes a universe emerging out of nothing without any tunneling. Also the largest peak in $|\Psi(u)|^{2}$ corresponds to the maximum of the scale factor in the classical oscillatory solution as shown in figure 1. 


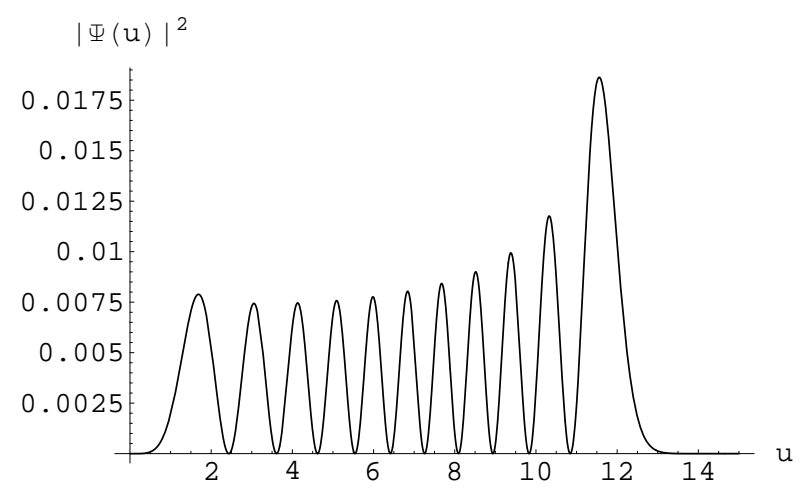

Figure 4: Square of the wavefunction for $M=g=1, \omega=0.025$ and $n=15$.

\section{Conclusions}

In this paper we have studied the properties of the Einstein-Dirac equations in a spatially flat Robertson-Walker background, with an eye to the signature changing solutions. We have shown that if the cosmological constant is negative, either free or self-interacting spinor fields result in solutions which admit a degenerate metric in which the scale factor and the physical quantities of interest such as scalar curvature and spinor field energy density have a continuous behavior in passing from a Euclidean to a Lorentzian domain. This phenomenon also appears when we have a self-interacting massless spinor field. The corresponding cosmology in these cases show an unbounded universe in the Euclidean region passing smoothly through the signature changing hypersurface and an oscillatory behavior in the Lorentzian domain. If the cosmological constant is positive or zero the signature changing solutions do not exist. In particular, equations (39-42) show a universe with a positive cosmological constant for which although signature transition occurs, the energy density becomes negative which does not correspond to ordinary matter. We have also shown that a massless free spinor field does not result in such solutions, irrespective of the choice of the potential.

The quantum cosmology of the model presented above and the ensuing WD equation is amenable to exact solutions in terms of Hypergeometric functions. These solutions correspond to the classical signature changing solutions and would be of interest in the context of the boundary condition of the universe.

Acknowledgement The authors would like to thank the research council of Shahid Beheshti university for financial support.

\section{References}

[1] Hartle J B and Hawking S W 1983 Phys. Rev. D 282960

Hawking S W 1984 Nucl. Phys. B 239257

Halliwell J J and Hartle J B 1990 Phys. Rev. D 411815

Gibbons G W and Hartle J B 1990 Phys. Rev. D 422458

[2] Ellis G, Sumeruk A, Coule D and Hellaby C 1992 Class. Quantum Grav. 91535

Ellis G 1992 Gen. Rel. Grav. 241047

Hayward S A 1992 Class. Quantum Grav. 91851

Martin J 1995 Phys. Rev. D 526708 [gr-qc/9509053]

[3] Dereli T and Tucker R W 1993 Class. Quantum Grav. 10365 
[4] Ghafoori-Tabrizi K, Gousheh S S and Sepangi H R 2000 Int. J. Mod. Phys. A 151521 [grqc/9910020]

[5] Dereli T, Önder M and Tucker R W 1993 Class. Quantum Grav. 101425

[6] Jalalzadeh S, Ahmadi F and Sepangi H R 2003 JHEP 08 (2003) 012 [hep-th/0308067]

[7] Embacher F 1995 Phys. Rev. D 522150 [gr-qc/9410012]

[8] Darabi F and Sepangi H R 1999 Class. Quantum Grav. 161565 [gr-qc/9905008]

[9] Zhlnorovich V A 2004 J. Exp. Theor. Phys. 98619 [gr-qc/0010039]

Saha B 2004 Phys. Rev. D69 124006 [gr-qc/0308088]

Armendariz-Picon C and Greene P B 2003 Gen. Rel. Grav. 351637 [hep-th/0301129]

Isham C J and Nelson J E 1974 Phys. Rev. D 103226

[10] Forger M and Römer H 2004 Annals Phys. 309306 [hep-th/0307199]

[11] Abramowitz M and Stegun I A 1972 Handbook of Mathematical Functions Dover, NewYork 